\title{
Diverting Ostomies and their Complications in Mechanical Colorectal Anastomosis for Rectum Cancer: Retrospective Study
}

\section{Rektum Kanserinde Mekanik Kolorektal Anastomozlarda Saptırıcı Ostomiler ve Komplikasyonları: Retrospektif Çalışma}

\author{
Bahadır Öndeş ${ }^{1}$, Niyazi Karaman², Bülent Aksel ${ }^{2}$, Cihangir Özaslan ${ }^{2}$ \\ ${ }^{1}$ Ercis Devlet Hastanesi, Genel Cerrahi Kliniği, Van, Türkiye \\ ${ }^{2}$ Ankara Onkoloji Eğitim Araştırma Hastanesi, Genel Cerrahi Kliniği, Ankara, Türkiye
}

Dergiye Ulaşma Tarihi: 10.03.2019 Dergiye Kabul Tarihi: 30.07.2019 Doi: 10.5505/aot.2019.26213

\section{ÖZET}

GİRIŞ ve AMAÇ: Anastomoz kaçağı aşağı anterior rezeksiyon sonrası en ciddi komplikasyondur ve morbidite mortalite oranlarını ve hastane kalış süresini uzatır. Bu çalışmada hastanemiz serisinde saptırıcı ostomilerin kolorektal anastomozlarda etkinlik ve güvenliğinin retrospektif olarak karşılaştırılması amaçlanmıştır.

YÖNTEM ve GEREÇLER: Hastanemizde 2005-2010 yılları arasında rektum adenokanseri tanısılla elektif olarak opere edilerek ekstraperitoneal kolorektal veya koloanal anastomoz yapılıp saptırıcı ostomi açılmış tüm hastaların dosyaları geriye dönük olarak incelendi.

BULGULAR: Çalışmaya toplam 101 hasta alınmıştır. Hastaların 29'una (\%28.7) neoadjuvan kemoradyoterapi (NAKRT) uygulanmıştı. Çalışma grubundaki hastaların 33'üne (\%32.7) saptırıcı ostomi uygulanmıştı. Bu hastaların 31'ine $(\% 93,9)$ ileostomi, ikisine ise kolostomi uygulanmıştı. NAKRT uygulanmış 29 hastanın 14'üne (\%48.2) saptırıcı ostomi açıldığı ve bunun da istatiksel olarak anlamlı olarak yüksek olduğu saptandı ( $\mathrm{p}=0.03)$. BMI $<25$ olan hastalardan sadece ikisine saptırıcı ostomi açılırken; BMI $\geq 25$ olanların 31 'ine saptırıcı ostomi açılmışı ve aradaki bu fark istatiksel olarak anlamlı bulundu $(\mathrm{p}=0.0001)$. Cerrahi alan enfeksiyonu 24 hastada (\%23.8) saptanan en sık komplikasyondu. Stoma açılan 33 hastanın 23'ünde (\%69.6) stoma ilişkili komplikasyonların geliştiği gözlendi. Saptırıcı ostomi açılan hastaların dördünde (\%12.2) dehidratasyon bulguları nedeniyle ek tedaviler uygulandığı saptandı. Parastomal cilt infeksiyonlarının ileostomi açılan 31 hastanın 14'ünde gözlendi (\%45).

TARTIŞMA ve SONUÇ: Bu hasta serisinde, NAKRT uygulanan ve BMI $\geq 25$ olan hastalarda saptırıcı ostomi uygulama sıklığının yüksek olduğu saptanmıştır. Primer cerrahi sonrası en sık komplikasyon olarak cerrahi alan infeksiyonu 24 hastada (\%23.8) saptanmıştır. Bu hasta serisinde en sık rastlanan stoma komplikasyonları siklık sırasıyla; deri irritasyonları (\%42.4), dehidratasyon $(\% 12,1)$, stoma kanaması (\%6.1), mükokütanöz ayrışma (\%6.1) ve stoma revizyonu (\%3) ihtiyacıdır. Saptırıcı ostomi uygulanan hastalarda bu komplikasyonlar açısından dikkatli olunmalıdır.

Anahtar Kelimeler: Rektum kanseri, aşağı anterior rezeksiyon, saptırıcı ostomi, morbidite, mortalite

\begin{abstract}
INTRODUCTION: Anastomotic leakage after low anterior resection is the most serious complication and increases the morbidity and mortality rates and prolonges length of stay. In this study, the effectiveness and safety of diverting ostomies in colorectal anastomosis was compared retrospectively. METHODS: The charts of all patients with diverting ostomies operated with extraperitoneal colorectal or coloanal anastomosis electively due to rectal adenocarcinoma between 2005-2010 were examined retrospectively. RESULTS: A total of 101 patients were included in the study. Twentynine (28.7\%) patients were given neoadjuvant chemoradiotherapy (NAKRT). Diverting ostomy was applied to 33 of the patients (32.7\%) in the study group. Diverting ileostomy was performed to $33(93.9 \%)$ of these patients, colostomy in the remaining two. Diverting ostomy was applied to 14 of 29 patients $(48.2 \%)$ treated with NAKRT $(\mathrm{p}=0.03)$. While the diverting ostomy was applied in 31 patients with BMI $\geq 25$, it was used only in two patients with BMI $<25(\mathrm{p}=0.0001)$. Surgical site infection was the most frequent complication in 24 patients (23.8\%). Stoma related complications were observed in 23 patients $(69.6 \%)$. Additional treatments for dehydration were needed in $4(12.2 \%)$ patients. Parastomal skin infections were observed in 14 of 31 patients $(45 \%)$ with ileostomy DISCUSSION AND CONCLUSION: In this series of patients, the diverting ostomy applications were found to
\end{abstract}


be more frequent in patients with BMI $\geq 25$ and treated with NAKRT. In this series of patients, the most common stoma complications were; skin irritations (42.4\%), dehydration (12.1\%), stoma bleeding (6.1\%), mucocutaneous dehiscence $(6.1 \%)$ and the need for stoma revision $(3 \%)$. In terms of these complications care should be taken in patients undergoing diverting ostomy.

Keywords: Rectum carcinoma, low anterior resection, diverting ostomy, morbidity, mortality

\section{GİRIŞ}

Anastomoz kaçağı aşağı anterior rezeksiyon sonras1 en ciddi komplikasyondur ve morbidite (reoperasyon, perkutan drenaj, septik komplikasyonlar, anastomoz stenozu) mortalite oranlarını ve hastane kalış süresini uzatır (1). Kaçak sıklığı rezeksiyon düzeyi, işlem tipi ve cerrahi deneyime bağl1 olarak \%2 - \%39 arasında bildirilmektedir (2).

Anastomoz kaçağı için risk faktörleri; erkek cinsiyet, anastomozun yüksekliği, preop RT ve ameliyat sirasindaki istenmeyen olaylar olarak sıralanabilir. Saptırıcı ostomiler sıklıkla geçici olarak, kaçakların etkilerini azaltmak ve kaçak ile ilişkili yeni girişim oranlarını azaltmak amaciyla uygulanırlar. Stomalar rahatsızlık, peristomal enflamasyon, dehidratasyon gibi birtakım morbiditeler oluştururlar. Kapatılması için ikinci ameliyat gerekir ve bunun da azımsanmayacak komplikasyonları mevcuttur.

Erken gelişen komplikasyonlar; barsağın karın içine kaçması, evisserasyon, iskemi ve stoma nekrozu, stoma retraksiyonu, kanama, parastomal enfeksiyon ve delinme, cerrahi alan enfeksiyonları, yüksek akışlı loop ileostomi, erken obstrüksiyon ve mukokutanöz ayrılmadır. Geç gelişen komplikasyonlar ise; stoma prolapsusu, parastomal fitıklar, stoma stenozu, stoma fistülü, peristomal cilt problemleri, geç dönemde barsak tıkanması, metabolik komplikasyonlar, üriner ve safra taş1 oluşumudur (3).

Stoma kapat1lmas1 \%2-60 morbidite ve \%0-5 mortaliteye yol açan bir girişimdir. Yara infeksiyonu ve anastomoz kaçakları komplikasyonların başında gelir. Erken kapatılan vakalarda morbidite daha da yüksektir. Lokalize küçük anastomoz kaçakları konservatif tedavi ile yara enfeksiyonları drenaj ve lokal bakımla kontrol altına alınır (4). Gerekirse bir süre total parenteral beslenme uygulanır. Anastomoz kaçağının en sık nedeni kötü kanlanma ve anastomoz hattındaki gerginliktir. Stomanın erken kapatılması ile postoperatif komplikasyon oranı yükselir. Altıbin hastalık bir seride \%17.3 kapama ilişkili morbidite bildirilmiştir (5). Hemen tüm serilerde stoma kapatılmasına bağl1 erken (nekroz, kanama) ve geç (fistül, infeksiyon, kesi fitığı, stoma prolapsusu gelişimi) komplikasyonların ve stomaya bağlı reoperasyonun loop kolostomi grubunda daha fazla olduğu bildirilmektedir $(6,7)$. Buna karşın yaşam kalitesi açısından değerlendirme yapıldığında lup kolostomi uygulanan hastalarda, lup ileostomi uygulananlara göre daha iyi yaşam kalitesi sonuçları bildirilmiştir

Rektal kanser cerrahisi sirasinda adhezyon bariyerleri kullanılarak saptırıc1 ileostominin 4-6 hafta içerisinde kapatılmasının KT uygulanmasinda gecikmelere neden olmaksızın mümkün olabileceği de bildirilmiştir (8). Stomaların kalıcı olarak kalma riski bazı çalışmalarda \%19'lara kadar yüksek olarak bildirilmiştir.

\section{HASTALAR VE METOD}

$\mathrm{Bu}$ çalışmada hastanemiz serisinde saptırıcı ostomilerin kolorektal anastomozlarda etkinlik ve güvenliğinin retrospektif olarak karşılaştırılması amaçlanmıştır. Hastanemizde 2005-2010 yılları arasında rektum adenokanseri tanisiyla elektif olarak opere edilerek ekstraperitoneal kolorektal veya koloanal anastomoz yapılıp saptırıcı ostomi açılmış tüm hastaların dosyaları geriye dönük olarak incelendi. Bu çalışma ile hastanemiz serisinde; saptırıc1 ostomi seçiminde ileostomi ve kolostomi karşılaştırılması, anastomoz kaçak sıklığı, anastomoz kaçağı saptanan hastalarda erken postoperatif ikincil girişimler, ostomi morbiditeleri, kapatılabilme oranları, kapatılamama nedenleri, kapatılma süreleri, kapatılmaya ilişkin komplikasyonlar ve bunlara yönelik ikincil cerrahi girişim ihtiyacı ve hastane kalış süreleri bilgilerine ulaşılmıştır.

Devamlı değişkenler için Student's t testi kullanıld1. Veriler retrospektif olarak topland, sayısal veriler için unpaired t-test ya da Mann-Whitney U-test, Nominal veriler için Chi-square test ya da Fisher's exact test kullanıldı. $\mathrm{Bu}$ amaçla ticari olarak satın 
alınabilen SPSS 16.0 statistical software programları kullanıldı.

\section{BULGULAR}

Çalışmaya toplam 101 hasta alınmıştır. Hastaların 59' u erkek, 42'si kadındır. Çalışma grubundaki hastaların yaş ortancası $61.1 \pm 13.2$ (14-88) olarak saptanmıştır. Tümörlerin anal girimden ortalama uzaklıkları $8.89 \pm 2.8 \mathrm{~cm}$ (3$15 \mathrm{~cm}$ olarak) saptand. Hastaların 29'una (\%28.7) neoadjuvan kemoradyoterapi (NAKRT) uygulanmıştı. Çalışma grubundaki 79 hastaya $(\% 78,2)$ aşağ 1 anterior rezeksiyon uygulanırken; 22 hastaya daha aşağı anterior rezeksiyon uygulanmıştı. Çalışma grubundaki hastaların 33'üne (\%32.7) saptırıcı ostomi uygulanmıştı. $\mathrm{Bu}$ hastaların 31'ine $(\% 93,9)$ ileostomi, ikisine ise kolostomi uygulanmıştı. NAKRT grubundaki hastalarda ameliyata kadar ortalama 8.9 hafta (6-12 hafta) beklendiği saptand. NAKRT uygulanmış 29 hastanın 14'üne (\%48.2) saptırıcı ostomi açıldığı ve bunun da istatiksel olarak anlamlı olduğu saptand $1(\mathrm{p}=0.03)$. BMI $<25$ olan hastalardan sadece ikisine saptırıcı ostomi açılırken; BMI $\geq 25$ olanların 31'ine saptırıcı ostomi açılmıştı ve aradaki bu fark istatiksel olarak anlamlı bulundu $(\mathrm{p}=0.0001)$. Saptırıc1 ostomilerin ortanca $6 \pm 3$ ay (1-12) sonra kapatılabildiği saptand. Cerrahi alan enfeksiyonu 24 hastada (\%23.8) saptanan en sik komplikasyondu. Ostomi Komplikasyonları Tablo 1'de özetlenmiştir. Saptırıcı ostomi açılmayan ve sonrasında anastomoz kaçağı saptanan 6 hastanın $(\% 8,9)$ beşine tedavi amaçlı saptırıcı ostomi uygulandı. Bu hastaların 4'ü (\%66) erkekti ve 5'i (\%83.3) ise NAKRT uygulanmayan grupta idi. Saptırıc1 ostomi açılan grupta kaçak tespit edilmemiştir. Hastalar ortalama 32 ay takip edildi. Hastaların 4'ünde (\%12) saptırıcı ostomiler takip süresinde kapatılamamıştı. $\mathrm{Bu}$ hastaların üçünün $(\% 75)$ kaybedilmiş olması nedeniyle ostomileri kapatılamamıştı. Stoma açılan 33 hastanın 23'ünde (\%69.6) stoma ilişkili komplikasyonların geliştiği gözlendi. Saptırıcı ostomi açılan hastaların dördünde (\%12.2) dehidratasyon bulguları nedeniyle ek tedaviler uygulandığı saptandı. İki hastada(\%6) gelişen mükokütanöz ayrışma basit sütürasyon ile onarıld1. Parastomal cilt infeksiyonlarının ileostomi açılan 31 hastanın 14'ünde gözlendi (\%45). Stoma kenarından oluşan kanama iki hastada (\%6) gözlendi ve kanayan vasküler oluşum sütür ile kontrol altına alınabildi. Stoma retraksiyonu nedeniyle bir hastaya (\%3) ostomi bölgesinden cerrahi girişim ile stoma revizyonu uygulanmas1 gerekti.

\begin{tabular}{lll}
\multicolumn{3}{l}{ Tablo 1. Ostomi Komplikasyonları } \\
\hline KOMPLİKASYON & SAYI & YÜZDE \\
\hline Deri İrritasyonları & 14 & $\% 42.4$ \\
\hline Dehidratasyon & 4 & $\% 12,1$ \\
\hline Stoma Kanaması & 2 & $\% 6.1$ \\
\hline $\begin{array}{l}\text { Mükokütanöz } \\
\text { Ayrışma }\end{array}$ & 2 & $\% 6.1$ \\
\hline Stoma Revizyonu & 1 & $\% 3$
\end{tabular}

Tablo 2. Hastaların demografik özellikleri ve ameliyat karakteristikleri

\begin{tabular}{ll}
\hline Hasta Bilgisi & \\
\hline Yaş & $61.1 \pm 13.2(14-88)$ \\
\hline Erkek & $59(58.4)$ \\
\hline Kadın & $42(41.6)$ \\
\hline BMI & $25.8 \pm 2.80(21.1-34.4)$ \\
\hline Ortalama Takip Süresi & $32 \pm 1.2(1-72$ ay $)$ \\
\hline $\begin{array}{l}\text { Tümörün Anal Kanala } \\
\text { Mesafesi }\end{array}$ & $8.89 \pm 2.8 \mathrm{~cm}(3-15 \mathrm{~cm})$ \\
\hline NAKRT Uygulanan & $29(\% 28.7)$ \\
& \\
\hline LAR/ULAR & $79(\% 78.2) / 22(\% 21.8)$ \\
\hline Ameliyat Süresi & $177 \pm 44.4 \mathrm{dk}$ \\
\hline İleostomi & $31(\% 93.9)$ \\
\hline Kolostomi & $2(\% 6.1)$ \\
\hline $\begin{array}{l}\text { Orta Hat Kesi İle } \\
\text { Kapat1lma }\end{array}$ & $7(\% 21.2)$ \\
\hline $\begin{array}{l}\text { Ostomi Bölgesinden } \\
\text { Kapatılma }\end{array}$ & $22(\% 78.8)$ \\
\hline
\end{tabular}

\section{TARTIŞMA}

$\mathrm{Bu}$ hasta serisinde tüm saptırıcı ostomiler elektif şartlarda açılmıştır. Literatürde, acil şartlarda stoma açılmasının komplikasyon riskini artırdığ 1 belirtilmektedir $(7,9)$. Del Pino ve ve ark. yaptığı çalışmada acil ameliyata alınan olgularda görülen komplikasyon sıklığının istatiksel olarak anlamlı düzeyde yüksek olduğu bildirilmektedir (10).Yine 
serimizde 31 hastaya ileostomi (\%93.9) ve sadece 2 hastaya (\%6.1) kolostomi açılmıştır. $\mathrm{Bu}$ nedenle gruplar arasında karşılaştırma yapılamamıştır. Ancak literatürde geçici ileostomilerle geçici kolostomileri karşılaştıran birçok çalışma mevcuttur (11-13). 1986 yılında Rutegard ve ark. çalışmalarında stoma ile ilgili komplikasyon oranın iki stoma grubu arasında anlamlı bir farklılık olmadığını ancak ileostomi ile ilgili komplikasyonların daha ciddi olduğunu belirtmişlerdir (11). Bizim serimizde yaklaşık \%70 hastada stoma ilişkili komplikasyonlar geliştiği gözlenmiştir. $\mathrm{Bu}$ komplikasyonlar arasında \% 42 oranı ile cilt irritasyonları en s1k olanıdır. Bunu \%12 oranı ile dehidratasyon takip etmektedir. Köckerling ve ark. çalışmalarında 464 hastanın retrospektif değerlendirilmesi sonucunda ileostomi grubunda cerrahi tedavi gerektiren komplikasyonların daha fazla olduğunu tespit etmişlerdir (12). Gooszen ve ark. yaptıkları randomize çok merkezli bir çalışmada ileostomi grubunda komplikasyonların ve cilt irritasyonunun beklenenden daha sık olduğunu saptamışlardır. Kolostomi grubunda da prolapsus daha sik bulunmuştur (13).

İleostomili hastaların 2/3'ünde günlük g1da ve sıvı alımının dengelenmesinin gerektiği, ileostomi kapatılmasinın kolostomi kapatılmasından daha sik ve daha ciddi komplikasyonlara yol açtığı belirtilmiştir. Ayrica barsak hasarlanması ve stenoz riskinin olduğu da vurgulanmıştır. Bizim serimizde deri irritasyonları da komplikasyon olarak kabul edildiğinde, yaklaşı $\% 70$ hastada stoma ilişkili komplikasyonlar geliştiği gözlenmiştir. Stoma morbiditesinin \%10-60 arasında değiştiği birçok kaynakta bildirilmektedir (14). Oranların bu denli farklı oluşu, bazı araştırmacıların peristomal deri irritasyonunu komplikasyon olarak kabul etmeyişinden ileri gelmektedir. Bazıları çok basit uygulamalarla ortadan kaldırılabilen bu komplikasyonların erken dönemde fark edilmeleri oldukça önemlidir (15). Duchesne ve ark. çalışmasında malignitenin stoma komplikasyonlarının üzerine etkisinin olmadığı bildirilmektedir (16). Literatürde bu konuda farklı fikirler bulunmaktadır. İleostomili ve kolostomili olgulardaki komplikasyon oranının literatürde geniş bir dağılımda olduğu görülmektedir (17). $\mathrm{Bu}$ oran \%10 ile \%70 arasında değişmektedir (15). Literatürde stoma türünün komplikasyonlar üzerine etkisi olduğunu bildiren birçok çalışma mevcuttur (18-20). Stoma komplikasyonları ile ilgili çalışmaların çoğunda ileostomi olgularında komplikasyon sıklığının daha fazla olduğu bildirilmektedir (20). Az sayıda çalışmada da kolostomili olgularda daha sik komplikasyon görüldüğü bildirilmektedir (6). İleostomili olgularda komplikasyon oranının \%40 ile \%90 arasında, kolostomili olgularda ise $\% 30$ ile $\% 70$ arasinda olduğunu belirten değişik çalışmalar vardır (2021).

\section{SONUÇLAR}

$\mathrm{Bu}$ hasta serisinde, NAKRT uygulanan ve BMI $\geq 25$ olan hastalarda saptırıc ostomi uygulama sıklığının yüksek olduğu saptanmıştır. Primer cerrahi sonrası en s1k komplikasyon olarak cerrahi alan infeksiyonu 24 hastada (\%23.8) saptanmıştır. Bu hasta serisinde en sik rastlanan stoma komplikasyonları sıklık sırasıyla; deri irritasyonları (\%42.4), dehidratasyon $(\% 12,1)$, stoma kanaması (\%6.1), mükokütanöz ayrışma (\%6.1) ve stoma revizyonu (\%3) ihtiyacidır. Saptırıc1 ostomi uygulanan hastalarda bu komplikasyonlar açısından dikkatli olunmalıdır. Rektum kanserinin cerrahi tedavisinde saptıric1 ileostomi uygulanmas1 bazı morbiditeleri de beraberinde getirmektedir. Ancak bu morbidite, rektum düzeyinde bir anastomoz kaçağının yol açabileceği daha ciddi morbiditeyi dengelemektedir.

\section{Çıkar Çatışması: Yok}

\section{REFERANSLAR}

1. X. Complications of colorectal anastomoses: leaks, strictures, and bleeding. Davis B(1), Rivadeneira DE. Surg Clin North Am. 2013 Feb;93:61-87. doi: 10.1016/j.suc.2012.09.014.

2. X1. Incidence and mortality of anastomotic dehiscence requiring reoperation after rectal carcinoma resection. Cong ZJ, Hu LH, Xing JJ, Bian ZQ, Fu CG, Yu ED, Li ZS, Zhong M. Int Surg. 2014 Mar-Apr; 99(2):112-9. doi: 10.9738/INTSURG-D-13-00059.

3. X2. Ostomy-Related Complications. Murken DR, Bleier JIS. Clin Colon Rectal Surg. 2019 May; 32(3):176-182. doi: 10.1055/s-0038-1676995.

4. Brown J.Y. The value of complete physiological rest of the large bowel in the treatment of certain ulcerative and obstructive lesions of this organ. Surg Gynecol Obstet 1913; 16:610-613.

5. Chow A, Tilney HS, Paraskeva P, Jeyarajah S, Zacharakis E, Purkayastha S. The morbidity surrounding reversal of defunctioning ileostomies: a systematic review of 48 studies including 6,107 cases. International journal of colorectal disease. Jun; 2009 24(6):711-723.

6. Rullier E, Le Toux N, Laurent C, Garrelon JL, Parneix M, Saric J. Loopileostomy versus loop colostomy for 
defunctioning low anastomoses during rectal cancer surgery. World J Surg 2001; 25:274-278.

7. Güenaga KF, Lustosa SA, Saad SS, Saconato H, Matos D. Ileostomy or colostomy for temporary decompression of colorectal anastomosis. Cochrane Database Syst Rev. 2007;24:CD004647.

8. Memon S, Heriot AG, Atkin CE, Lynch AC. Facilitated early ileostomy closure after rectal cancer surgery: a casematched study. Tech Coloproctol. 2012 Aug;16(4):28590.

9. Stothert JC Jr, Brubacher L, Simonowitz DA. Complications of emergency stoma formation. Arch Surg 1982;117:307-309.

10. Del Pino A, Cintron JR, Orsay CP, Pearl RK, Tan A, Abcarian H. Enterostomal complications: are emergently created enterostomas at greater risk? Am Surg 1997; 63:653-656.

11. Rutegard J, Dahlgren S. Transvers colostomy or loop ileostomy as diverting stoma in colorectal surgery. Acta Chir Scand 1986;153:229-232.

12. Köckerling F, Parth R, Meisner M, Hohenberger W. Ileostomie-Koecalfistel Kolostomie-Welches ist das geeignetste vorgeschaltete Stuhlableitungsverfahren unter Berücksichtigung von Angle, Funktion, Komplikationen und Rückverlagerung? Zentalb Chir 1997; 122:34-38.

13. Gooszen AW, Geelgerken RH, Hermans J, Lagaay MB, Gooszen HG. Temporary decompression after colorectal surgery: randomized comparision of loop ileostomy and colostomy. Br J Surg 1998; 85:76-79.

14. Kodner IJ. Intestinal Stomas. In Zinner JM, Swartz SI,(Eds) Maingot's Abdominal Operation, 10 thEd, pp.427-460, Appleton-Lange Stanford, 1997

15. Alemdaroğlu K, Akçal T, Buğra D. Kolon Rektum ve Anal Bölge Hastalıkları. Türk Kolon ve Rektum Cerrahisi Derneği, 2. Bask1: 309-329, İstanbul, 2004.

16. Duchesne JC, Wang YZ, Weintraub SL, Boyle M, Hunt JP; Stoma complications: a multivarite analysis. Am Surg 2002; 68:961-966.

17. Flikier-Zelkowicz B, Codina-Cazador A, Farrés-Coll R, Olivet-Pujol F, MartínGrillo A, Pujadas-de Palol M. Morbidity and mortality associated with diverting ileostomy closures in rectal cancer surgery. Cir Esp. 2008 Jul;84(1):16-9.

18. Daly JM, De Cosse JJ. Complications in surgery of the colon and rectum. Surg Clin North Am 1983; 63:12151231.

19. Porter JA, Salvati PA, Rubin RJ, Eisenstat TE: Complications of Colostomies. Dis Colon Rectum 1998; 32:299-303.

20. Pearl RK, Prasad ML, Orsay CP, Abcarian H, Tan AB, Melz MT: Early local complications from intestinal stomas. Arch Surg 1985;120:1145-1147.

21. Moscowitz DA, Kirschner JB: Ileostomy in ulcerative colitis. Am J Surg 1994;141:727-729. 